\title{
Implementation of a Hybrid Stabilizing Controller on a Mobile Robot with Two Degrees Of Freedom
}

\author{
Wilco Oelen ${ }^{1}$ \\ ${ }^{1}$ University of Twente \\ Mechatronics Research Centre Twente \\ P.O. Box 217, 7500 AE Enschede \\ The Netherlands \\ ${ }^{3}$ University of Twente \\ Department of Applied Mathematics \\ P.O. Box 217, 7500 AE Enschede \\ The Netherlands
}

\author{
Henk Nijmeijer ${ }^{3} \quad$ Carlos Canudas de $\mathrm{Wit}^{4}$ \\ ${ }^{2}$ Hollandse Signaalapparaten B.V. \\ P.O. Box 42 \\ 7550 GD Hengelo \\ The Netherlands
${ }^{4}$ Laboratoire d'Automatique de Grenoble BP 46
38402 Saint-Martin d'Hères, Grenoble
France

\begin{abstract}
One of the results of a research project on mechatronics is a mobile robot, which was used as a carrier for research on the design of mechatronical systems. This mobile robot, the Mobile Autonomous Robot Twente (MART), is intended to be used as major part of a future assembly factory.

In this paper the implementation on the MART-robot of a recently developed hybrid controller for stabilization of non-holonomic systems is described. Limitations of the physical model of the mechanical structure of the MARTrobot lead to a number of practical problems. An interesting result given in the paper is the insight obtained into the geometrical meaning of different parameters of the controller, which allowed considerable improvements in the performance of the controller on the actual mobile robot.
\end{abstract}

\section{Introduction}

Stabilization of non-holonomic systems to an arbitrary point in state space in general is quite difficult. An example of a non-holonomic system is a car, which can be steered to any position and any orientation in free space. At the same time the freedom of motion of a car is limited; it cannot move sideways. Possible applications of nonholonomic stabilizing control might be in industrial environments, where mobile robots have to visit specific work stations, or in automated parking.

Many practical non-holonomic systems, such as mobile robots, cars and trucks with one or more of trailers can be modelled as nonlinear systems in chained form [5][8] (see also section 2). Because of their practical importance the stabilization of such systems has received much attention in recent literature. Stabilization cannot be achieved by smooth static state feedback [2]. For this reason other solutions have been proposed, based on for instance timevarying feedback [4][6][7], discontinuous feedback [8] and hybrid feedback [3]. In most of these publications, the usefulness of the approaches is demonstrated by computer simulations. However, because such simulation studies usually neglect such practical aspects as nonlinear system dynamics, rolling friction and compliance of the mechanical structure, their outcomes are of limited value. Moreover in our opinion the final justification for these research efforts lies in the practical implementation.

To the best of our knowledge, this paper offers the first reported empirical verification of a stabilizing controller for non-holonomic systems. For this purpose, the hybrid control concept [3] was implemented on a mobile robot with two degrees of freedom (2 DOF), which can be described as a one-chained, nonlinear system [3]. This experimental robot system has two driven wheels and a castor to carry the mechanical structure. The robot was designed as part of the MART-project, a research project on the design of complex mechatronical systems.

Special attention is paid to a number of controller implementation issues. One of these issues is that the hybrid controller is designed for a system with velocity input ('kinematics'), whereas in practice we have to deal with torque or force input ('dynamics'). Hence, a velocity controller was developed that transforms the mobile robot to a new system with two velocity inputs: linear velocity and angular velocity. The hybrid stabilizing controller is implemented on this velocity-controlled mobile robot. Another issue is the selection of controller design 
parameters. Experiments provided us useful insight into the physical meaning of different controller components. This insight allowed us to significantly increase the control performance.

In the next section, 2, the hybrid controller as proposed by Canudas de Wit et al. [3], is applied to the stabilization of a 2-DOF mobile robot. In section 3 the hardware and practical setup of the mobile robot is discussed. Section 4 covers the implementation of the hybrid controller on the velocity-controlled mobile robot. In section 5 experimental results are shown of the stabilized mobile robot. Section 6 contains some concluding remarks and a number of recommendations on the hybrid controller and on the mechanical structure.

\section{Hybrid stabilization of a mobile robot}

The controller presented here consists of two parts. A discrete-time part that stabilizes the error in the driving direction and a piecewise continuous-time part that stabilizes the lateral error. This controller is based on a general controller for $n$-dimensional, nonlinear systems in one-chained form [3]. The kinematics of the mobile robot can be transformed to 3-dimensional, one-chained form by means of a coordinate transformation. In order to take into account dynamical limitations of the mechanical structure, the one-chained form, derived from the kinematics of the mobile robot, is extended by two integrators in a special way.

\subsection{Transformation to chained form}

A 2 DOF mobile robot can be described by

$$
\begin{aligned}
& \dot{x}=v \cos (\varphi) \\
& \dot{y}=v \sin (\varphi) \\
& \dot{\varphi}=\omega .
\end{aligned}
$$

Here $x, y$ and $\varphi$ are the position and orientation of the vehicle and $v$ and $\omega$ are the linear and angular velocity of the vehicle (see also section 3 ). The kinematics can be transformed locally to one-chained form, using the following coordinate transformation, which is intended to be used for values of $\varphi$ in the interval $\langle-1 / 2 \pi, 1 / 2 \pi\rangle$.

$$
\begin{aligned}
& x_{1}=x \\
& x_{2}=\tan (\varphi) \\
& x_{3}=y
\end{aligned}
$$

The transformation requires the inputs to be defined as

$$
\begin{aligned}
& u_{1}=v \cos (\varphi) \\
& u_{2}=\frac{\omega}{\cos ^{2}(\varphi)} .
\end{aligned}
$$

Using (2) and (3), the system transforms into a 3dimensional system with one chain [3], with states $x_{1}(t)$, $x_{2}(t)$ and $x_{3}(t)$ and inputs $u_{1}(t)$ and $u_{2}(t)$.

$$
\begin{aligned}
& \dot{x}_{1}=u_{1} \\
& \dot{x}_{2}=u_{2} \\
& \dot{x}_{3}=u_{1} x_{2}
\end{aligned}
$$

Subsystem (4a) can be stabilized by applying piece-wise constant control signals $u_{1}(t)$. During one time interval, in which $u_{1}(t)$ is constant, the remaining subsystem $(4 \mathrm{~b}, \mathrm{c})$ can be regarded as a time-invariant linear system with one input signal $u_{2}(t)$. This system is controllable as long as $u_{1}(t) \neq 0$, and in this case a continuous-time controller exists, that stabilizes $(4 \mathrm{~b}, \mathrm{c})$. The continuous time controller has to be designed, such that $x_{2}(t)$ and $x_{3}(t)$ are stabilized (much) faster than $x_{1}(t)$.

\subsection{Extension of one-chained system}

If a discrete-time controller is used for $x_{1}(t)$, then $u_{1}(t)$ has to change abruptly after every discrete sampling interval. This is not possible for a real mobile robot, because of its inertia. This problem can be solved by simply adding integrators in cascade to each of the inputs of the onechained system. In [3], however, integrators are added in a non-standard way:

$$
\begin{aligned}
& u_{1}(t)=\int_{0}^{t} v_{1}(\tau) \mathrm{d} \tau \\
& u_{2}(t)=\int_{0}^{t} v_{1}(\tau) \mathrm{d} \tau \int_{0}^{t} v_{2}(\tau) \mathrm{d} \tau
\end{aligned}
$$

The reason for adding the integrators in this particular way is that the extended system can be rewritten in chained form again, by introducing two new state variables, $w_{1}(t)$ and $w_{2}(t)$ :

$$
\begin{aligned}
& \dot{w}_{1}(t)=v_{1}(t) \\
& \dot{x}_{1}(t)=w_{1}(t) \\
& \dot{w}_{2}(t)=v_{2}(t) \\
& \dot{x}_{2}(t)=w_{1}(t) w_{2}(t) \\
& \dot{x}_{3}(t)=w_{1}(t) x_{2}(t) .
\end{aligned}
$$

Here $v_{1}(t)$ and $v_{2}(t)$ are the new inputs of the system. If they are piece-wise continuous in time, then $u_{1}(t)=w_{1}(t)$ and $u_{2}(t)=w_{1}(t) w_{2}(t)$ are continuous in time. Subsystem (6a) again is stabilized using a discrete-time controller and subsystem (6b) is stabilized using continuous-time control. 
If $v_{1}$ is piecewise constant during time intervals $\mathbf{I}_{\mathbf{k}}=$ $[k \delta,(k+1) \delta\rangle$, where $\delta$ is the sample interval of the discretetime controller, then the discrete-time system

$$
\begin{aligned}
& w_{1}(k+1)=w_{1}(k)+\delta v_{1}(k) \\
& x_{1}(k+1)=x_{1}(k)+\delta w_{1}(k)+\frac{1}{2} \delta^{2} v_{1}(k)
\end{aligned}
$$

is an exact, discrete representation of (6a). Here $v_{1}(k), w_{1}(k)$ and $x_{1}(k)$ are shorthand notations for $v_{1}, w_{1}$ and $x_{1}$ at time $k \delta$.

\subsection{Geometrical interpretation}

To each of the quantities, introduced above, a geometrical interpretation can be given. For $x_{1}$ and $x_{3}$ this is exact. For the other quantities, except $u_{2}$, it is an approximation with an accuracy better than $7 \%$ as long as $|\varphi|<20^{\circ}$.

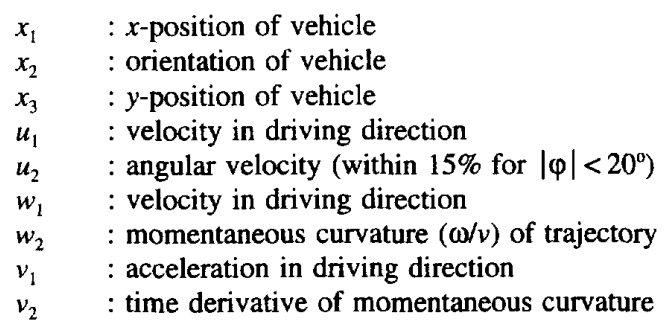

\subsection{Stabilization of extended one-chain system}

Here the hybrid controller is given for the specific case of the transformed kinematics of the mobile robot, extended with two integrators.

Consider the subsystems (7), and (6b), together with the following hybrid controller:

$$
\begin{aligned}
v_{1}(k)= & \mathrm{K}_{1}(\xi(k))+\mathrm{H}(\xi(k)) \alpha(\|z(k \delta)\|) \\
v_{2}(t)=- & \left|w_{1}(t)\right|\left(k_{z, 1} w_{2}(t)+\right. \\
& \left.\quad \operatorname{sign}\left(w_{1}(t)\right) k_{z, 2} x_{2}(t)+k_{z, 3} x_{3}(t)\right),
\end{aligned}
$$

where

$$
\begin{aligned}
& z(t)=\left[\begin{array}{lll}
z_{1}(t) & z_{2}(t) & z_{3}(t)
\end{array}\right]^{\mathrm{T}}=\left[\begin{array}{lll}
w_{2}(t) & x_{2}(t) & x_{3}(t)
\end{array}\right]^{\mathrm{T}} \\
& \xi(k)=\left[\begin{array}{lll}
w_{1}(k) & x_{1}(k)
\end{array}\right]^{\mathrm{T}},
\end{aligned}
$$

and $\mathrm{K}_{1}($.$) is any asymptotically stabilizing discrete-time$ feedback controller for subsystem (7) and

$$
\mathbf{H}(\xi(k))=\operatorname{sign}\left(w_{1}(k)+\frac{\delta}{2} \mathrm{~K}_{1}(\xi(k))\right) .
$$

$\alpha(r)$ is any continuous, strictly increasing function of $r$, with the property $\alpha(r) \rightarrow \infty$ if $r \rightarrow \infty$, and the positive constants $k_{\mathrm{z}, j}(1 \leq j \leq 3)$ are such that all zeros of

$$
\lambda^{3}+k_{\mathrm{z}, 1} \lambda^{2}+k_{\mathrm{z}, 2} \lambda+k_{\mathrm{z}, 3}
$$

have negative real part. Then the closed loop system (7), $(6 b),(8 a, b)$ is asymptotically stable [3].

In the implementation of this controller the parameters $k_{z_{j}}$ $(1 \leq j \leq 3)$ and the functions $\alpha($.$) and K_{1}($.$) have to be tuned$ in order to achieve an acceptable performance. This subject is covered in section 4 . The norm $|z(k)|$ may be any norm, but Canudas de Wit et al. [3] use an Euclidian norm for their simulation study.

\section{Mobile Autonomous Robot Twente}

The goal of the MART project is to gain insight in how to design complex mechatronic systems. This insight should be demonstrated by the design and realization of a specific system, the Mobile Autonomous Robot Twente. This project results from a preliminary study by Abrahams [1], who developed a concept for a future automated assembly hall. Instead of assembly of products on a conveyor belt, Abrahams proposed a more flexible and more robust system, where a set of mobile robots takes care of the assembly tasks. In Abrahams' concept, each mobile robot consists of a vehicle, which carries a manipulator. Assembly can be done on the vehicle (also during motion for increased throughput) and on fixed work stations. The concept of Abrahams allows the use of 2 DOF mobile robots, as long as they can be positioned sufficiently accurately.

\subsection{Mechanical structure of the mobile robot}

The vehicle is split in two parts. Both parts are connected to each other by means of soft springs. The lower part contains the wheels and the motors for driving the wheels. The upper part of the vehicle carries the manipulator and the batteries and contains the necessary electronics. The soft springs provide a good suspension, without the need of very soft tires. In figure 1 a schematic drawing of the mobile robot is given.

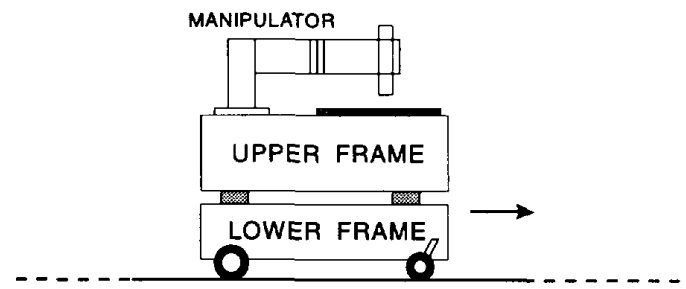

Figure 1. Schematic drawing of the MART-robot 
The lower part of the vehicle has a mass of $75 \mathrm{~kg}$. The upper part of the vehicle has a mass of $400 \mathrm{~kg}$. The dominant resonance frequency of the suspended upper frame is $3 \mathrm{~Hz}$ in all directions. The length of the vehicle is $1.4[\mathrm{~m}]$, its width is $1.0[\mathrm{~m}]$ and its height is $0.8[\mathrm{~m}]$. The lower frame has two driven wheels and one castor. The castor limits the steering capabilities, because of coulomb friction. This is especially true when the castor flips from forward to backward orientation or vice versa.

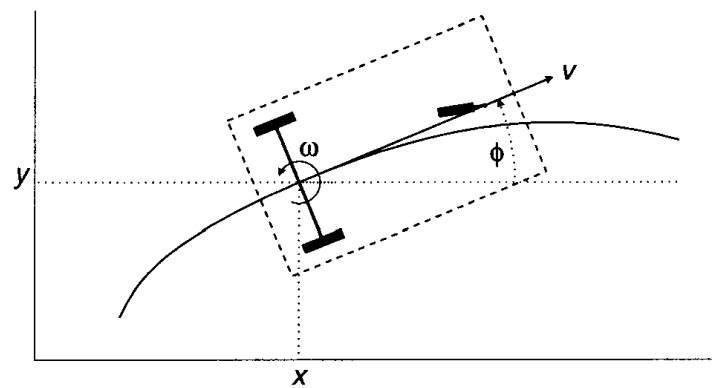

Figure 2. Wheel configuration of lower frame

The linear velocity in the driving direction is denoted by $v$ the angular velocity is denoted by $\omega$. The point $(x, y)$ corresponds to the point halfway between the two driven wheels on the rear axis. The orientation of the vehicle, $\varphi$, is measured relative to the $x$-axis of the world coordinate system. The kinematics of the vehicle are given in (1).

\subsection{Velocity control}

The hybrid stabilizing controller is designed for a system with velocity inputs. The mobile robot, however, has two torque inputs. This problem is overcome by applying velocity control to the mobile robot. A simple planar dynamical model is used for compensation of coulomb friction in the driven wheels and of centripetal forces. Friction in the castor is not compensated. A linear feedback law is superimposed on the compensation.

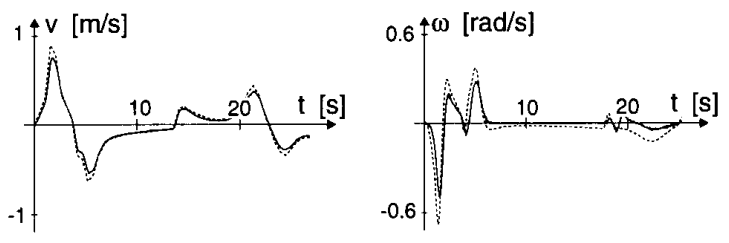

Figure 3. Reference and measured $v$ and $\omega$

Figure 3 illustrates the performance of the velocitycontrolled vehicle. Both the reference signals (dashed lines) and the measured actual signals are given. Both control inputs were applied simultaneously. Linear velocity tracking of the velocity-controlled vehicle is much better than angular velocity tracking. This is due to uncompensated friction in the castor.

\subsection{Position control}

The system, with velocity controller, can be regarded as a new system, with transfer $I+\Delta$ from $v_{\mathrm{d}}$ and $\omega_{\mathrm{d}}$ to $v$ and $\boldsymbol{\omega}$, where $I$ is a unity transfer and where $\Delta$ is a perturbation term, due to the imperfect velocity controller. Specific $v(t)$ and $\omega(t)$ result in a certain $x(t), y(t)$ and $\varphi(t)$, determined by the kinematics of the vehicle. The hybrid controller can be built around the velocity-controlled system $I+\Delta$ :

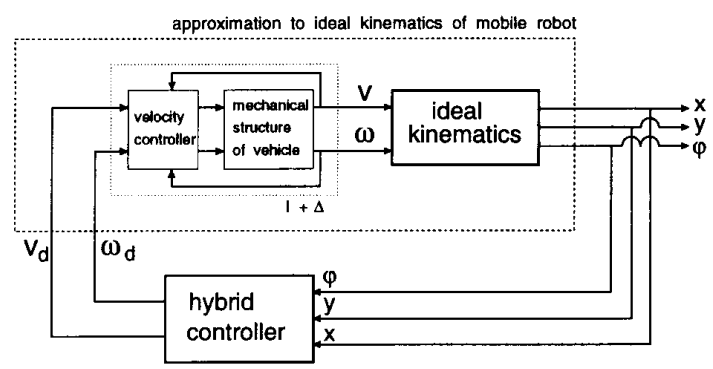

Figure 4. Structure of position controlled system

\subsection{Practical setup of the mobile robot system}

The hybrid controller is implemented in OCCAM on a T800 transputer, running at $17.5 \mathrm{MHz}$. On the same transputer the velocity controller and some I/O processes (file $\mathrm{I} / \mathrm{O}$, screen output and $\mathrm{AD} / \mathrm{DA}$ conversion) are running. The vehicle is connected to an 80386 host $\mathrm{PC}$ by means of a $10 \mathrm{Mbit} / \mathrm{s}$ transputer link. The controllers run at a sampling rate of $300 \mathrm{~Hz}$. This is well above the main resonance frequencies of the mechanical structure, which are $3 \mathrm{~Hz}$ and $20 \mathrm{~Hz}$.

The vehicle is placed in a room of approximately $6 \times 6$ $\left[\mathrm{m}^{2}\right]$. This limits the allowable excursions of the vehicle to approximately $2[\mathrm{~m}]$ around its initial position, which is in the middle of the room.

Measuring the position and orientation of the vehicle is done by integrating wheel revolutions (odometry).

\section{Implementation of the hybrid controller}

In the implementation of the hybrid controller a stabilizing linear feedback with constants $a_{1}$ and $a_{2}$ is chosen for $\mathrm{K}_{1}$ (.). A quadratic function, multiplied by a constant $\alpha_{0}$, is chosen for $\alpha($.$) (see section 2.4):$ 


$$
\begin{aligned}
& K_{1}(\xi(k))=a_{1} x_{1}(k)+a_{2} w_{1}(k) \\
& \alpha(r)=\alpha_{0} r^{2} ; \quad r^{2}=\|z(k)\|^{2}=w_{2}(k)^{2}+x_{2}(k)^{2}+x_{3}(k)^{2}
\end{aligned}
$$

Here a short hand notation $\mathbf{z}(k)$ is introduced for $\mathbf{z}(k \delta)$. A similar notation is used for the elements of $\mathbf{z}(k \delta)$.

The error in driving direction is stabilized by the discrete time controller $\mathrm{K}_{1}($.$) . The lateral error and orientation error$ are stabilized by the continuous-time controller (8b). The second term of the discrete-time control signal, $v_{1}(k)$ (see section 2.4), introduces a disturbance in the driving direction if there is a lateral error or an orientation error. The resulting controller has the following parameters:

$\delta$

$k_{z, j}(1 \leq j \leq 3)$

$a_{1}$ and $a_{2}$

$\alpha_{0}$

duration of the time interval during which $v_{1}(t)$ is constant coefficients of characteristic polynomial of lateral error dynamics coefficients of discrete-time characteristic polynomial of dynamics in the driving direction

gain at which lateral and orientation errors force the vehicle to move again in the driving direction

The larger the parameter $\delta$, the longer the excursions made by the vehicle. The parameter $\delta$ was tuned such that the excursions fit safely in the available room. Parameters $k_{z_{j}}$ $(1 \leq j \leq 3)$ determine the three poles of the continuous-time subsystem with feedback. The lowest resonance frequency of the velocity-controlled vehicle is approximately $3 \mathrm{~Hz}$, i.e. $20 \mathrm{rad} \mathrm{s}^{-1}$. The magnitude of the poles has to be much lower. They are chosen at -6 and $-2.5 \pm j 1.5$. Parameters $a_{1}$ and $a_{2}$, together with $\delta$, determine the two poles of the discrete-time subsystem with feedback $K_{1}($.$) . These poles$ are chosen such that the error in driving direction converges much slower than the lateral and orientation errors. Below follows a list of all parameter-values:

$$
\begin{aligned}
& \delta=0.5 ; \alpha_{0}=1 ; a_{1}=-0.36 ; a_{2}=-1.11 ; \\
& k_{\mathrm{z}, 1}=11 ; k_{\mathrm{z}, 2}=38.5 ; k_{\mathrm{z}, 3}=51
\end{aligned}
$$

In the practical setup, where exact converge is impossible, the vehicle stops if the following margins are satisfied:

$$
\begin{aligned}
& |x|<0.01[\mathrm{~m}] \\
& |y|+c|\varphi|<0.10[\mathrm{~m}] \quad c=1\left[\mathrm{~m} \mathrm{rad}^{-1}\right] \\
& |v|<0.02\left[\mathrm{~m} \mathrm{~s}^{-1}\right] \\
& |\omega|<0.02\left[\mathrm{rad} \mathrm{s}^{-1}\right]
\end{aligned}
$$

\section{Experimental results}

In the first experiments an Euclidian norm was used for $\|z(k)\|$ (see section 2.4). In later experiments the norm was modified. This modification is based on insight in the physical meaning of $|z(k)|$. This increased the performance of the controlled vehicle considerably.

\subsection{Experiments using Euclidian error-norm}

If there are only initial errors in driving direction and as long as the initial castor orientation is aligned properly with the vehicle's driving direction then the controller behaves well. Experiments with a non-zero initial lateral error show less satisfactory results. In most test runs the vehicle moved forward and backward without end, even with the large error bounds given in section 4 . This is due to friction in the castor.

\subsection{Modification of error-norm}

By retuning the parameters of section 4 , the behaviour of the controlled system could be made slightly better, but no real improvements were obtained. The disturbance in driving direction, due to lateral errors should be stronger, while the disturbance, due to orientation errors should remain the same. This disturbance is introduced by means of the function $\alpha(\|z(k)\|)$, see (8a). In the initial setup an Euclidian norm was used for $|z(k)|$, as was used by Canudas de Wit et al. [3] in their simulation studies:

$$
\|z(k)\|=\sqrt{w_{2}(k)^{2}+x_{2}(k)^{2}+x_{3}(k)^{2}}
$$

The coordinate $x_{3}(k)$ equals the lateral error $y(t)$ of the vehicle, at time $k \delta$. In order to selectively increase the coupling between lateral error and motion in driving direction, the error-norm $\mid z(k) \|$ is redefined:

$$
\|z(k)\|_{\text {new }}=\sqrt{w_{2}(k)^{2}+x_{2}(k)^{2}+\alpha_{y} x_{3}(k)^{2}}
$$

A new parameter $\alpha_{y}$ is introduced, which is chosen equal to 3. All other parameters remain the same. After this modification the vehicle reaches its end goal within a reasonable number of excursions.

\subsection{Experiments with modified error-norm}

In figure 5 a run is shown, with an initial lateral error of 0.3 [m]. The covered trajectory is shown in the $x y$-plane.

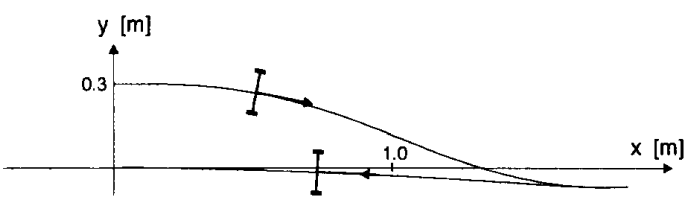

Figure 5. Trajectory of vehicle in $x y$-plane 
Note the length of the excursion. It is approximately 1.8 [m] in $x$-direction. After approximately 15 seconds the vehicle has reached its end goal, within a few $\mathrm{cm}$. Another interesting feature is the control signal $v_{1}(k)$. Because of the initial non-zero lateral error the vehicle accelerates in the driving direction. Once the lateral error and orientation error are compensated well enough $v_{1}(k)$ converges to zero. Switching can be seen every half second. The control signal $v_{1}(k)$ is plotted in figure 6 , together with $x(t)$. The vertical axis ranges from $-2[\mathrm{~m}]$ to $+2[\mathrm{~m}]$ for $x(t)$ and from $-1\left[\mathrm{~ms}^{-2}\right]$ to $+1\left[\mathrm{~ms}^{-2}\right]$ for $v_{1}(k)$.

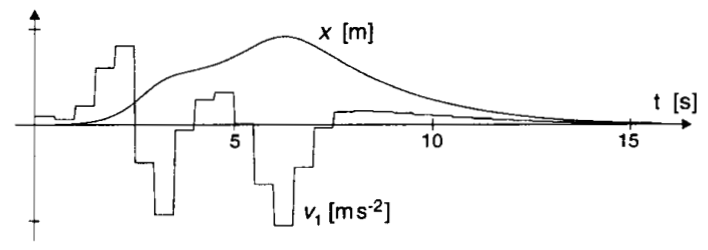

Figure 6. $v_{1}(k)$ and $x(t)$ as function of time

For compensation of the lateral error sufficiently long excursions should be made. This effect is demonstrated very well by another test run. During this run the vehicle moves forward and backward several times. First a number of excursions are made with a total length of less than 1 $[\mathrm{m}]$ in the driving direction. As soon as there is a longer excursion (total length approximately 1.5 [m]), the lateral error can be compensated within the error margins. The results of this run are given below.

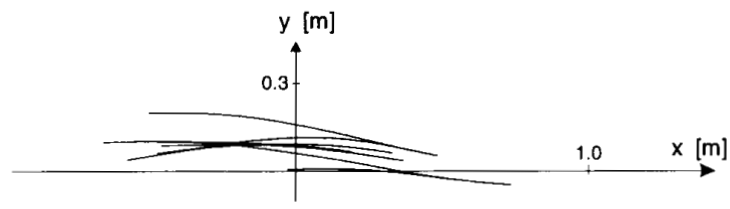

Figure 7. Test run with initial errors $x=-0.5$ [m], $y=0.2[\mathrm{~m}], \varphi=0$ [rad]

Figure 7 shows the path of the mobile robot in the $x y$-plane and figure 8 shows $x$ and $y$ as functions of time.

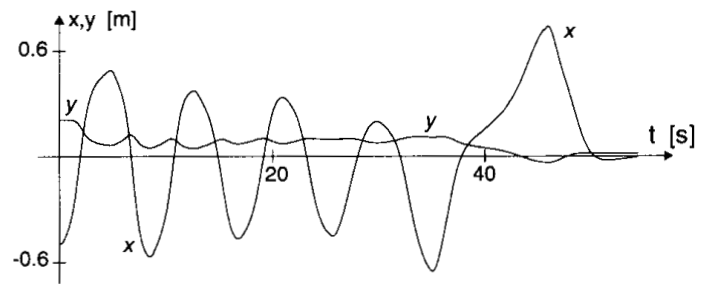

Figure 8. Test run with $x, y$ as functions of time

\section{Conclusions}

A hybrid controller, developed for non-holonomic systems, has been successfully implemented on a real mobile robot with two degrees of freedom. By using a geometrical interpretation of the quantities appearing in the controller the performance was increased considerably.

The controller performance suffers from steering problems, because of significant castor friction. During stabilization of the vehicle, the driving direction changes intermittently. The castor needs a fairly large distance to flip from one direction to the other direction, so the vehicle should move in the same direction for a sufficiently long distance.

The controller performs better if longer excursions of the vehicle are allowed during stabilization. This can be achieved by increasing the discrete sampling interval of the controller or by making the dynamics of the discretetime part of the controlled system slower (i.e. making the dynamics in the driving direction slower).

\section{References}

[1] Abrahams J. (1985), Zeer voorlopig onderzoek naar flexibele assemblage systemen (in Dutch), CFT Philips, Eindhoven, The Netherlands

[2] Brockett, R.W. (1983), Asymptotic stability and feedback linearization, Differential Geometric Control Theory, pp. 181-191, Birkhäuser.

[3] Canudas de Wit, C., H. Berghuis, and H. Nijmeijer (1993), Hybrid stabilization of Nonlinear Systems in Chained Form (preprint, to be submitted).

[4] Coron, H.-M., and J.-B. Pomet (1992), A remark on the design of time-varying stabilizing feedback laws for controllable systems without drift, Proc. IFAC Symp. Nonlinear Control Systems Design, pp. 413-417, Bordeaux, France.

[5] Murray, R.M., and S.S. Sastry (1991), Steering nonholonomic systems in chained form, Proc. IEEE Conf. on Decision and Control, pp. 1121-1126, Tucson, Arizona

[6] Pomet, J.-B. (1992), Explicit design of time-varying stabilizing control laws for a class of controllable systems without drift, Systems and Control Letters, vol. 18, pp. 147-158

[7] Samson, C. (1990), Velocity and torque feedback control of a non-holonomic cart, Int. Workshop on Adaptive and Nonlinear Control: Issues in Robotics, Grenoble, France, Nov. 21-23, 1990, pp. 125-151. Proc. in Advanced Robot Control, Vol. 162, Springer Verlag, C. Canudas de Wit (Ed).

[8] Sørdalen, O.J. (1993), Conversion of the kinematics of a car with $n$ trailers into a chained form, IEEE Conf. on Robotics and Automation, Atlanta, Georgia 